\title{
SARS-CoV-2-Pandemie - medizinische Entscheidungshilfe wegen der Gefahr drohender Ressourcenknappheit
}

\author{
Franz Ploner
}

Die COVID-19-Pandemie stellt die nationalen und internationalen Gesundheitssysteme vor immense Herausforderungen. Aufgrund der Geschwindigkeit, mit der das Coronavirus (SARS-CoV-2) sich ausgebreitet hat, ist eine außerordentliche Lage entstanden, die zu einem Massenzustrom von Patienten mit der Erkrankung COVID-19 in die Notaufnahmen, die, insbesondere in den Krankenhäusern des norditalienischen Raumes, durch sogenannte Prä-Triagezelte zur Verhinderung der Ausbreitung von Infektionen in den Kliniken ausgestattet sind, geführt hat.

In den Notaufnahmen, den Intensivstationen und den Abteilungen der Akutkrankenhäuser bestand deshalb die Befürchtung, die Behandlungsmöglichkeiten für die Patienten und Patientinnen durch die sich anbahnende Ressourcenknappheit einschränken zu müssen und nach einem Triagierungssystem die Verteilung und Prioritätensetzung den veränderten klinischen Bedingungen anpassen zu müssen. Die italienische Gesellschaft für Anästhesie, Reanimation und Intensivmedizin (SIARRTI) hat aus diesem Grund ein Positionspapier unter dem Titel Klinisch-ethische Empfehlungen für den Einsatz und das Aussetzen intensivmedizinischer Behandlungen unter den außergewöhnlichen Umständen und Bedingungen eines Ungleichgewichts zwischen Bedürfnissen, Nachfrage und Ressourcen verfasst, das Empfehlungen zum Umgang mit Patienten enthält und angesichts des gravierenden Mangels an medizinischen Ressourcen Zuweisungskriterien beinhaltet, nach welchen die Patienten mit den höchsten Chancen auf therapeutischen Erfolg Zugang zur Intensivmedizin erhalten können. ${ }^{1}$ Die Zuweisung der Ressourcen auf den Intensiv- und Subintensivstationen ist eine komplexe und heikle Aufgabe. Die Entscheidungskriterien für die Aufnahme und Entlassung unter diesen ungewöhnlichen Umständen der Ressourcenknappheit muss flexibel sein und sich vor allem an die lokalen Situationen der einzelnen Zentren anpassen. Das 13 Punkte umfassende Strategiepapier des SIARRTI versucht, Empfehlungen zu den einzelnen Aspekten in der Vorgangsweise bei der Betreuung der Patienten in den Notaufnahmen

1 Vgl. SIARRTI: Raccomandazioni di etica. 
und den Intensivstationen unter dem Blickwinkel der knappen medizinischen und strukturellen Ressourcen im Rahmen dieser COVID-19Pandemie zu geben. ${ }^{2}$

Gerade in den norditalienischen Krankenhäusern bestand die Befürchtung, dass nicht nur eine Rationalisierung der knappen Ressourcen notwendig werden könnte, sondern eine medizinische Rationierung der vorhandenen Mittel eintreten könnte mit der Gefahr, dass nicht mehr alle Patienten die medizinischen Leistungen erhalten würden, die ihnen entsprechend der italienischen Verfassung diritto alla tutela della salute zustehen müsste und worauf sie nach dem principio di uguaglianza e dovere di solidarietà ein Anrecht hätten. In einer solchen zeitlich begrenzten Ausnahmesituation, die den Zeitraum zwischen Februar und April 2020 umfasste, war es unabdingbar, dass alle Entscheidungen der involvierten Personen nach transparenten Regeln getroffen wurden und auf der Basis medizinethischer und moralischer Aspekte erfolgten. Basis der Entscheidungen bilden die vier weitgehend anerkannten medizin-ethischen Prinzipien (Gutes tun, Nichtschaden, Respekt vor der Autonomie und Gerechtigkeit), die gerade bei Ressourcenknappheit eine besondere Bedeutung haben. ${ }^{3}$ Die Abklärung des Patientenwillens, insbesondere bei jenen Personen, die einer Risikogruppe angehören, muss frühzeitig erfolgen. Knappe Ressourcen sollen nicht eingesetzt werden, wenn dies nicht dem Patientenwillen entspricht und ein Widerspruch zu einer vorliegenden Patientenverfügung besteht.

Norditalien wurde mit der größten COVID-19-Epidemie außerhalb Asiens, die mit der ersten nachgewiesenen Infektionskette in Lodi am 21. Februar 2020 begann, überrollt. ${ }^{4}$ Es herrschte, bedingt durch Berichte aus China, die Befürchtung, dass durch die sich anbahnende Epidemie relativ rasch eine Verknappung an Intensivbetten eintreten könnte. In Anbetracht der raschen Ausbreitung der Erkrankung war es notwendig, dass die Krankenhäuser und alle Organisationen im Gesundheitssystem der Region gemeinsam mit den politischen Entscheidungsträgern vorausschauend eine Strategie entwickelten, die eine Überforderung der Gesundheitsstrukturen verhinderte.

Aus diesem Grund wurde als Reaktion auf den COVID-19-Ausbruch in der Region Lombardei und nachfolgend in allen anderen Regionen und Provinzen des Landes Ende Februar und Anfang März 2020 eine regionale Task Force für alle Intensivbetten und COVID-Stationen eingerichtet, die

2 Vgl. SIARRTI: Raccomandazioni di etica.

3 Vgl. Carinci, Covid-19.

4 Vgl. Xie et al., Critical care crisis and some recommendations. 
die Governance-Richtlinien für die Notfallprozesse, die mit der COVID-19-Erkrankung in Zusammenhang standen, vorgab und die Verteilung der Ressourcen für alle Patienten mit COVID-19-Erkrankung zentral koordinierte. Die Task Force wurde entweder vom Leiter des Notfalldepartements und der Notfallnummer 112 oder dem regionalen Leiter der Intensivstationen geführt; ihnen zur Seite standen die Pflegedirektionen, die ärztlichen Leiter der einzelnen Krankenhäuser, das Management der Sanitätsbetriebe, die Leiter der virologischen Labors, die Leitung der Hygienedienste des Territoriums und der Krankenhäuser sowie ein Bioethiker. Die Task Force hatte primär zwei Schlüsselaufgaben zu erledigen:

a) die Erhöhung der Aufnahmekapazität positiv getesteter und schwer kranker COVID-Patienten in den Krankenhäusern und Intensivstationen durch Ausweisung von sogenannten COVID-Stationen und Aufstockung der Intensivplätze für die kritisch kranken Patienten sowie

b) die Ausarbeitung eines Konzeptes der strikten Eindämmung und Isolierung positiv getesteter Personen oder Verdachtsfälle.

Mehrere Arbeitsgruppen, die sich mit unterschiedlichen Fragestellungen befassten, arbeiteten entsprechende Empfehlungen für die einzelnen Bereiche aus und stellten diese den einzelnen Fachgruppen vor. Tägliche telemedizinische Life-Schaltungen zwischen der Task-Force-Gruppe und den Fachgruppen in den einzelnen Krankenhäusern erlaubten ein abgestuftes Aufnahmeverfahren der COVID-19-Patienten und die Koordination der freien Intensiv- und Abteilungsbetten nach dem Schweregrad der Erkrankung. Dadurch wurde versucht, die vorhandenen Ressourcen zu bündeln und die Überlastung des Gesundheitssystems so weit als möglich zu verhindern. Das gemeinsame Ziel sowohl der Task Force als auch der Geschäftsführung war es, die Patientenversorgung in diesem Ausnahmezustand zu garantieren und die Krankenhaussicherheit vor Infektionen durch das SARS-CoV-2-Virus zu gewährleisten. Ein wesentliches Ziel bestand zusätzlich darin, die physische und psychische Überbelastung der Mitarbeiter/-innen zu verhindern und ihnen gesundheitliche Sicherheit zu garantieren, um den Zusammenbruch des Gesundheitssystems durch zusätzliche Ausfälle an Mitarbeitern/-innen zu verhindern. Diese Zielvorgabe konnte am Höhepunkt der Pandemie in der Region Lombardei kaum garantiert werden.

Die aktuelle COVID-19-Pandemie ist eine völlig neue Herausforderung für unsere staatlich organisierten Gesundheitssysteme, sodass nicht auf bestehende Erfahrungswerte zurückgegriffen werden kann. Die gewonnenen Erfahrungen und Erlebnisse müssen aufgearbeitet und evaluiert werden, damit diese bei zukünftigen Entscheidungen zur Bewältigung einer sol- 
chen Pandemie in die Strategieplanung und die Organisation einfließen können.

\section{Literatur}

Carinci, Fabrizio: Covid-19: preparedness, decentralisation and the hunt for patient zero, in: https://doi.org/10.1126/bmj.m799 [09.06.2020].

SIARRTI: Raccomandazioni di etica per l'ammissione a trattamenti e per la loro sospensione, in condizioni eccecionali di squilibro tra necessità e risorse disponibili, 06.03.2020.

Xie, Jianfeng/Tong, Zhaohui/Guan, Xiangdong/Du, Bin/Qiu, Haibo/Slutsky, Arthur S.: Critical care crisis and some recommendations during the COVID-19 epidemic in China, in: https://doi.org/10.1007/s00134-020-05979-7 [09.06.2020]. 